\title{
Refractory cutaneous polyarteritis nodosa with threat of loss of feet: successful treatment with rituximab
}

\author{
Julie E. Stephan ${ }^{1}$ and David A. Minna ${ }^{2 *}$
}

*Correspondence: david.minna@utsouthwestern.edu

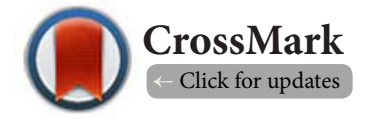

${ }^{1}$ MPAS, PA-C UT Southwestern Medical Center Dallas, Texas, USA.

${ }^{2} \mathrm{MD}$, FACR UT Southwestern Medical Center Dallas, Texas, USA.

\begin{abstract}
Cutaneous Polyarteritis Nodosa (CPAN) is a rare, single organ vasculitis of unknown etiology which presents with livedo reticularis and tender nodules involving the lower extremity in $97 \%$ of cases. Diagnosis is established by skin biopsy demonstrating segmental leukocytoclastic vasculitis and fibrinoid necrosis with workup excluding any systemic involvement (Figure 1). The presence of IgM antiphosphatidylserine-prothrombin complexes has led to the hypothesis that CPAN is a localized arthrus reaction. CPAN is characterized as a benign, chronic, relapsing vasculitis to be treated conservatively. As a single organ vasculitis, CPAN can affect arteries of any size. Due to CPAN's predilection for the lower extremities, involvement of the limb at or below the ankle's major arteries may result in loss of digits and potentially feet. The case presented involves CPAN in which the main arteries to the feet are occluded. The patient had been refractory to therapy with findings of early digital necrosis. Following adequate skin biopsy to establish a diagnosis, we initiated targeted therapy with rituximab, azathioprine, and high dose steroids with reversal of the ischemic process. It is the authors' opinion that CPAN should be treated more aggressively from initial diagnosis to prevent morbidity and potentially abort a chronic relapsing course.
\end{abstract}

Keywords: Cutaneous polyarteritis nodosa, rituximab therapy, refractory cutaneous polyarteritis nodosa, CPAN, cutaneous PAN

\section{Introduction}

The 2012 Chapel Hill Consensus Conference Nomenclature of Vasculitides defined Systemic Polyarteritis Nodosa (PAN) as a non-ANCA associated necrotizing arteritis of medium or small arteries. This delimitation of PAN makes it a rare disease (PAN: $31 / 10^{6}$, GPA: $160 / 10^{6}$, MPA: 94/10 ${ }^{6}$ ) [2] the single organ presentation of CPAN is rarer still. The true incidence is unknown [3] it has been estimated to be $<3 \%$ of all cutaneous vasculitides [4]. CPAN is characterized as a "benign, chronic, relapsing vasculitis" [5] requiring conservative management with NSAIDs, low dose steroids, and possibly met otrexate; as opposed to, the malignant course of systemic PAN which necessitates aggressive therapy with high dose steroids and immunosuppressive agents [1]. However, CPAN has been reported to cause distal extremity necrosis resulting in amputation $[6,7]$. When CPAN involves the distal lower extremity's intermediate size arteries, there is an increased risk of loss of digits and limb and aggressive therapy must be considered.
CPAN is a rare diagnosis which may be missed in favor of other more prevalent vasculitides. There are limited initial laboratory findings, while the clinical findings of tender nodules, livedo reticularis, and palpable purpura are associated with multiple diagnoses increases the probability ofa missed CPAN diagnosis. This may be compounded by insufficient biopsy material [3], especially with foot and ankle involvement.

Presented here is a case of CPAN in which bilateral vascular occlusion at the level of the ankles threaten loss of digits and potentially feet which reversed with combination therapy of high dose steroids, rituximab, and azathioprine.

\section{Case presentation}

The patient is a 38-year-old white female who presented to the outpatient rheumatology service with a ten month history of progressive bilateral foot pain. The right mid foot and forefoot were first involved, followed by the left ankle and hindfoot. Initially, no findings of swelling, temperature variation, skin 
discoloration or rash had been present. She had no history of cutaneous, pharyngeal, respiratory infections, new medications, or illicit drug use. At four weeks from onset of symptoms, she developed signs of ischemia, acral cyanosis, palpable purpuric lesions of the right forefootand left hind foot.

The patient returned for re-evaluation with a community rheumatologist two months after onset of symptoms with a workup including negative ANA, DNA, ENA, ANCA panel, hepatitis $B$ and $C$ serologies, and cryoglobulins. The patient's hypercoagulability profile was negative except for a positive phosphatidylserine IgM. From this data, a presumptive diagnosis of vasculitis had been made which prompted therapy ofprednisone $20 \mathrm{mg}$ and methotrexate $12.5 \mathrm{mg}$ weekly. The patient had been maintained on this regimen for six months with progression ofher symptoms. Subsequently, a skin biopsy from the right lateral foot demonstrated focal changes of leukocytoclastic vasculitis. The shallowness of the biopsy without biopsy of an artery limited diagnostic interpretation.

Admission to the hospital was later necessary due to further worsening of her symptoms. Upon admission, her vital signs were normal. Her Skin exam revealed the following: "dark and purple" feet with non-blanching, tender, purpuric lesions; right toes were cyanotic and tender to palpation; distal pulses were palpable but not rated. The remainder of her physical exam was within normal limits. Her labs were significant for a leukocytosis of 14.6, an anemia with hemoglobin of 10.7 , elevated platelet count of 599 , sedimentation rate of 30 (0-20), phosphatidylserine IgM of 50 (0-24), and an ANA of $1: 160$. The remainder of her serologies was unrevealing including DNA, ENA, complements, ANCA panel, hepatitis Band $C$ serologies, cryoglobulins, and her hypercoaguable profile. Of note, the patient also demonstrated a normal trans-esophageal echocardiogram and lower extremity doppler study for deep vein thrombosis. The patient underwent $\mathrm{CT}$ angiograms of her abdomen, pelvis, and bilateral lower extremities which revealed no abnormalities to the level of the ankles at which point the right and left anterior tibial and dorsalis pedis arteries abruptly taper or cut off. In addition, the left foot demonstrated the left posterior tibial artery to be the sole vascular supply to the left foot, the left anterior tibial artery occluded at the ankle, and the peroneal artery occluded in the distal calf. Similarly, the right foot shows no in-line flow to the foot, the posterior tibial artery occludes at the level of the calcaneous, the anterior tibial artery occluded at the ankle, and the peroneal artery supplies collaterals to the foot (Figures 2-5) Finally, the digital arteries are irregular, bilaterally, consistent with the diagnosis of vasculitis. In view of her diagnosis of vasculitis, she was discharged on $60 \mathrm{mg}$ prednisone daily and continued on methotrexate $12.5 \mathrm{mg}$ weekly.

One week after her discharge, the patient presented to our clinic. At this time, patient presented with livedo changes of bilateral feet, tender palpable purpuric lesions on the right medial forefoot and left medial hind foot, acral cyanosis of the right 1 st and 4th toes with early necrosis of the digital pulps
(Figures 6-8). Her dorsalis pedis pulses were non palpable bilaterally; posterior tibial pulses were palpable $2 / 4$ on left foot only. Her neurologic exam was within normal limits. Her labs again showed leukocytosis, thrombocytosis, anemia, elevated $c$ reactive protein, and normal urinalysis and creatinine. A skin biopsy was performed from the digital pulp of the right 1 st toe demonstrating an arteriole, inflamed by neutrophils, histiocytes, and lymphocytes with fibrin deposition in the muscular wall, and occlusion of lumen (Figure 1). This pattern is consistent with PAN.

Taken together, the objective evidence led to the establishment of a CPAN diagnosis. Accordingly, therapy was initiated with $60 \mathrm{mg}$ prednisone daily, azathioprine $100 \mathrm{mg}$ daily, and rituximab $1000 \mathrm{mg}$ day 0 and day 15 . After eight weeks from initiation of therapy, her diffuse foot pain had resolved and skin color improved, but patient had developed a painful 1 $\mathrm{cm}$ ulcer on tip of her right 1st toe (Figures 9 and 10).

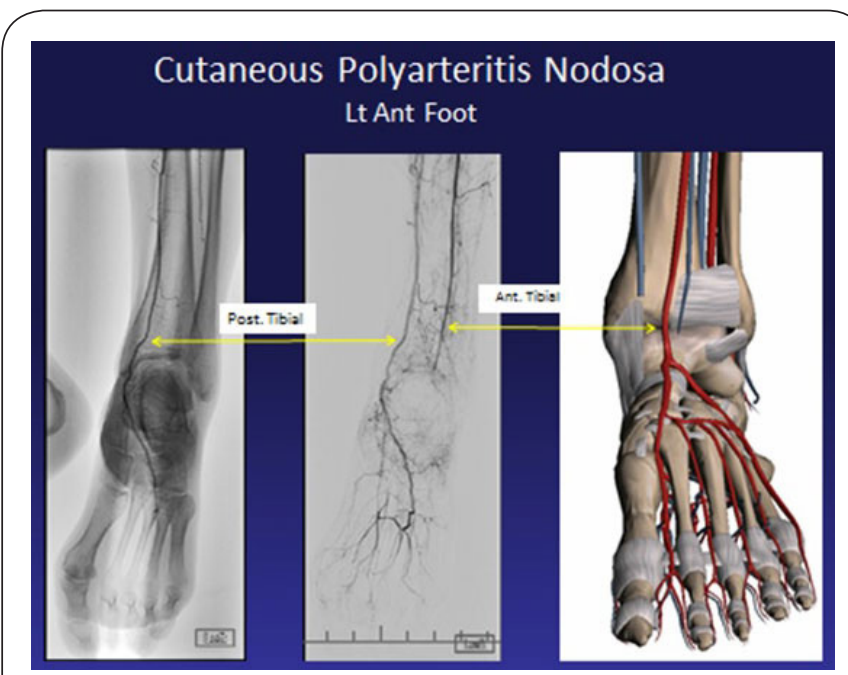

Figure 1. Skin biopsy with fibrinoid necrosis.

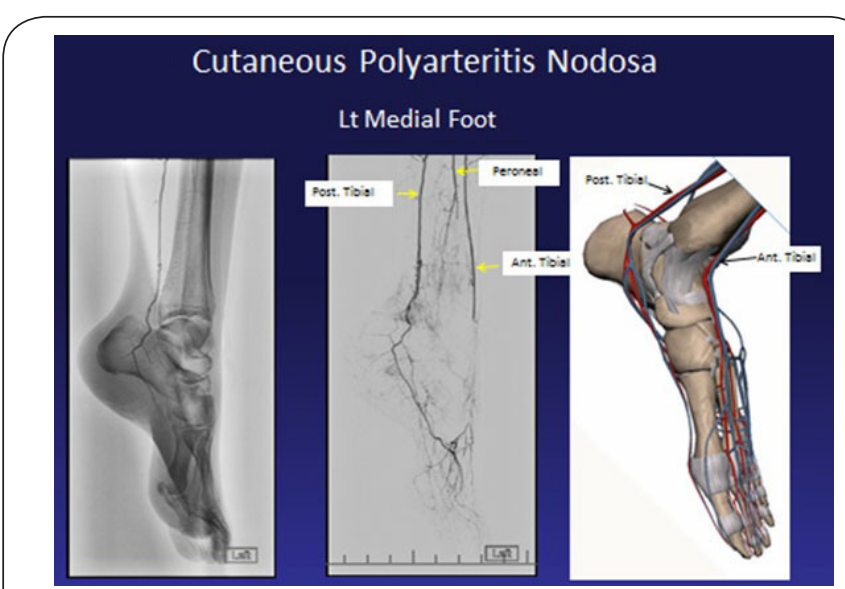

Figure 2. Lt Foot: Lt Post Tibial Artery is sole supply of lt foot. Lt ant-tibial artery occludes at ankle. Peroneal artery occludes in distal calf. Irregularity of all digital arteries consistent with dxvasculitis. 


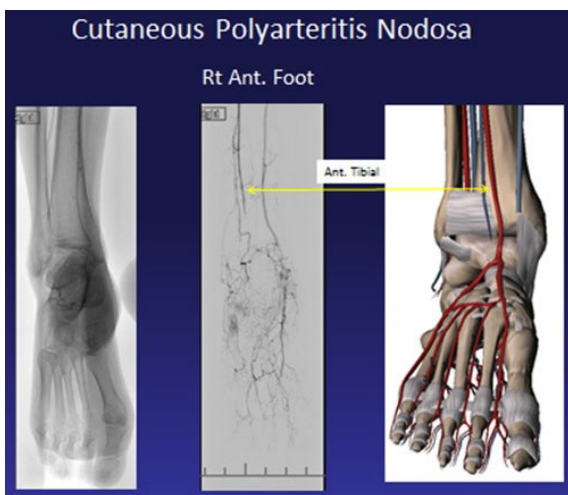

Figure 3. Lt Foot: Lt Post Tibial Artery is sole supply of lt foot. Lt ant-tibial artery occludes at ankle. Peroneal artery occludes in distal calf. Irregularity of all digital arteries consistent with dxvasculitis.

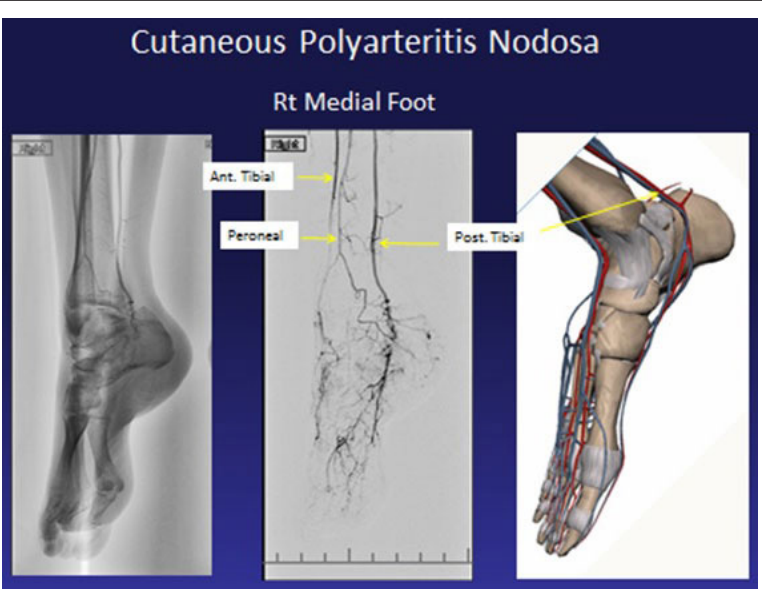

Figure 4. Rt Foot: There is no in-line flow to the Rt foot. Post tibial artery occludes at level of calcaneus. The ant tibial artery occludes at the ankle. Peroneal artery supplies collaterals to foot. Digital arteries are irregular consistent with $\mathrm{dx}$ of vasculitis.

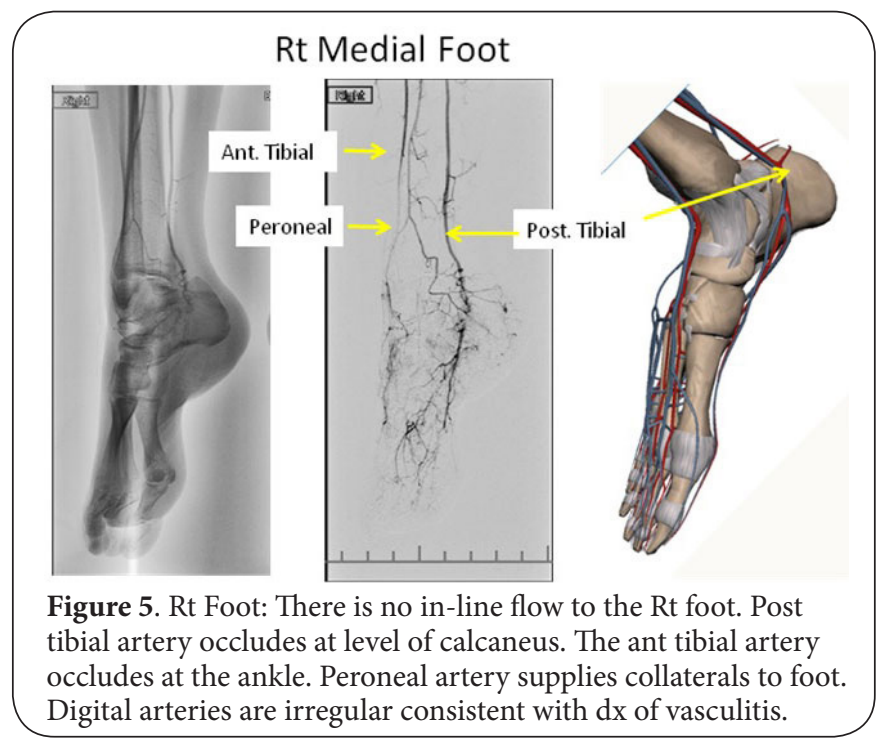

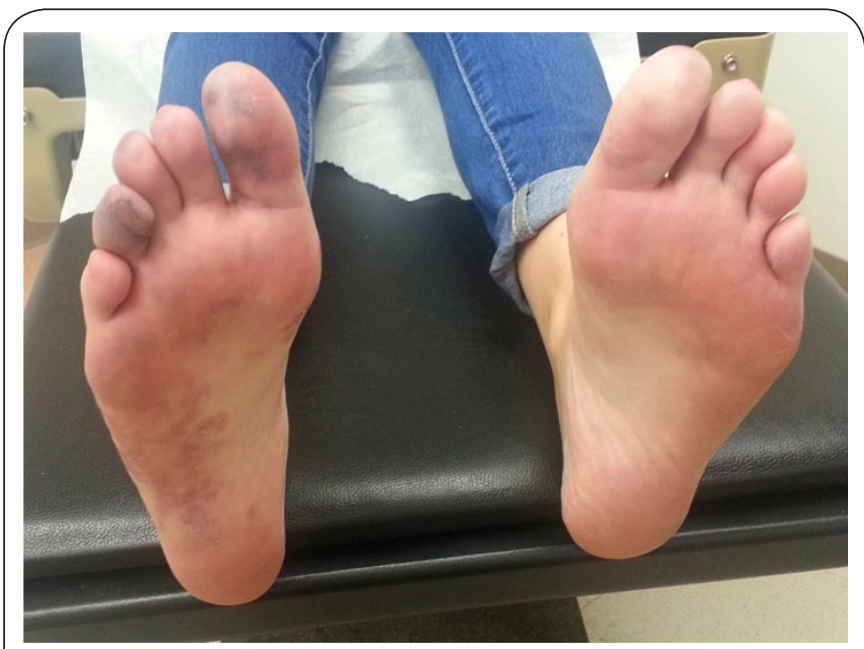

Figure 6. Feet Pretreatment.

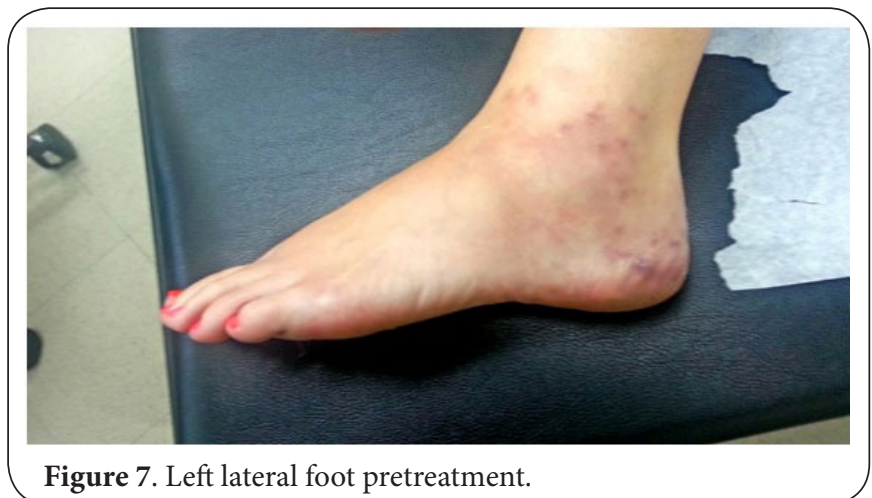

Figure 7. Left lateral foot pretreatment.

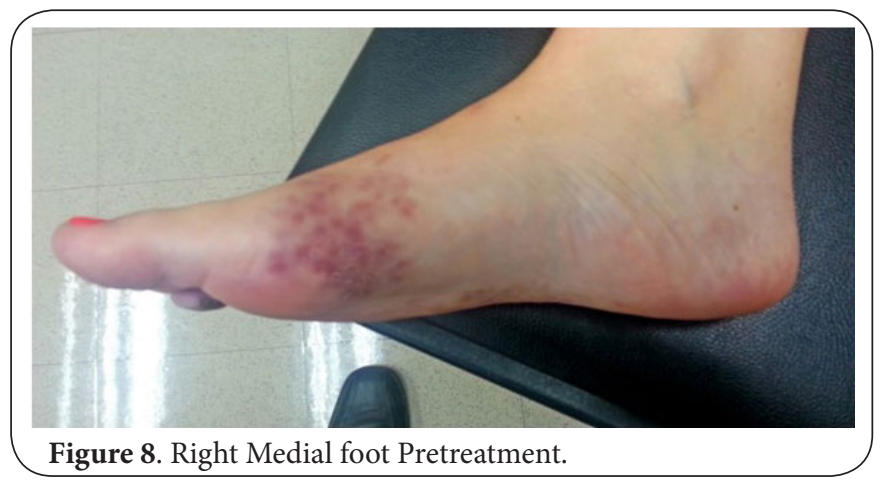

\section{Discussion}

Cutaneous Polyarteritis Nodosa, a rare single organ vasculitis, whose pathology is a segmental, necrotizing, leukocytoclastic vasculitis, may affect muscular arteries of any size. The initial manifestation is livedo reticularis which is also associated with antiphospholipid syndrome. Findings of CPAN do not fulfill criteria for antiphospholipid syndrome; however, antiphospholipid antibodies have been hypothesized to be involved in its pathogenesis. In one study, $81 \%$ of patients with CPAN 

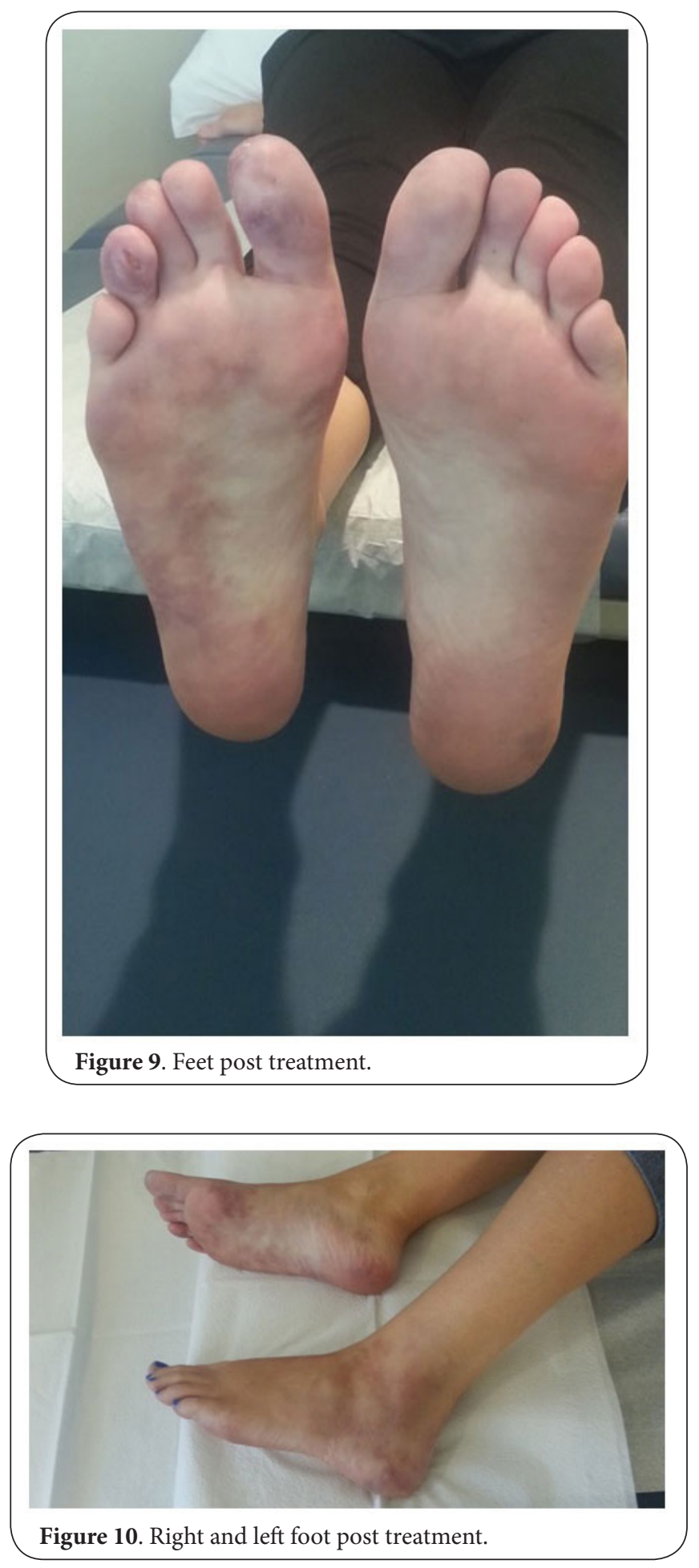

were reported to have IgM antiphophatidylserine-prothrombin complexes with all controls negative. The hypothesis is prothrombin bound to apoptotic endothelial cells induces anti-PS/ PT antibody production. These immunoglobulin complexes then activate the classical complement pathway causing CPAN. Direct immunofluorescence shows IgM deposition in vessel walls in $60 \%$ of specimens and C 3 in $40 \%$ of specimens with no deposits at the dermal-epidermal junction. It is postulated that a localized arthrus reaction is occurring in the vessel wall $[3,8]$. As a result, the chronic, relapsing course of CPAN is indicative of persistent antibody production and varying concentration of antigen. Therefore, the reduction of the inciting clone of $B$ cells would reduce immune complex formation to inhibit the inflammatory processand restrainthe chronic, relapsing course. In other words, the elimination of the B cell clone would prevent recurrence of CPAN.

The non-systemic pattern of involvement in CPAN is portrayed as a benign process warranting conservative management with nonsteroidals and/or colchicine, unless the disease proves to be refractory to conservative management $[3,5]$. Refractory disease is characterized by one or more of following: persistence of cutaneous lesions, severe pain in involved region, ulcerations, necrosis, fever, myalgias, arthralgias, dysesthesias, or neuropathy [3]. The reported prevalence of the aforementioned in CPAN cases is as follows: skin ulcers develop in $50 \%$, arthralgias $69 \%$, myalgias $31 \%$, neuropathy $22 \%$, and fever $25-30 \%$ [3]. At the time of diagnosis, refractory CPAN should be the expected disease course rather than the exception. It would be prudent and in the patient's longterm interest to institute an aggressive course of therapy unless contraindicated.

Symptom amelioration in $78 \%$ patients with CPAN has been reported with warfarin therapy [9]. Anticoagulation is premised on the presence of phosphatidylserine prothrombin complexes and antibodies with findings of thrombosis in cases of CPAN. Corticosteroid therapy in doses from $30 \mathrm{mg}$ to $1 \mathrm{mg} / \mathrm{kg}$ of prednisone is recommended in refractory cases; steroids control acute exacerbations of CPAN. Remissions have been reported, but upon tapering the steroid dose exacerbations of CPAN are generally reported [3]. Steroid sparing therapies reported for CPAN include hydroxychloroquine, dapsone, azathioprine, methotrexate, Ivlg, and cyclophosphamide [10-13]. Although immunosuppressives have been reported to be effective in CPAN, there are no controlled prospective trials to evaluate their efficacy. Use of immunosuppressives has been reserved for the most serious and unresponsive cases of CPAN [3]. Rituximab has been reported to be effective in a single case of CPAN with digital gangrene that was refractory to therapy with steroids and cyclophosphamide; no recurrence was reported at six months [14].

Our patient had occlusions of all lower extremity vessels save for the left posterior tibialis artery. She had been treated with steroids and methotrexate for six months with progression of her symptoms. Use of cyclophosphamide for this case was avoided since the use of cyclophosphamide in a 38 year old female may induce a premature menopause ( $>50 \%$ incidence) unless pre-medicated with leuprolide [15]. Considering the immediate threat of loss of digits, significant potential for loss of foot, and probable irreversibly compromised arterial supply to feet which future recurrences of CPAN would 
exacerbate, we decided to infuse rituximab $1000 \mathrm{mg}$ day 0 and day 15 with concomitant use of azathioprine $100 \mathrm{mg}$ and $60 \mathrm{mg}$ prednisone daily. Rituximab has been shown to be effective in other types of vasculitides for induction and maintenance therapy [16-18].

Eight weeks from her initial rituximab infusion, the patient had resolution of her diffuse bilateral foot pain, improvement of skin color, and improved nail bed perfusion. The cyanosis of her right $1^{\text {st }}$ and $4^{\text {th }}$ toes had resolved, but the right $1^{\text {st }}$ toe despite improved perfusion developed a $1 \mathrm{~cm}$ full thickness ulcer of the digital pulp at the base of digit. The patient was able to weight bear and walk without limitations. Her steroids have been tapered to $20 \mathrm{mg}$ daily and azathioprine continued. It is planned to repeat the rituximab dose of $1 \mathrm{gram}$ at day 0 and 15 at six months. Following her second cycle ofrituximab, therapy will be based from clinical observation. Since the presence of IgM phosphatidylserine-prothrombin (PST/ PT) complexes have been reported in $81 \%$ of patients with CPAN [8]; PST/PT complexes have been used as a diagnostic marker for CPAN, but have neither been studied in regards to disease activity, nor success of therapy. Assessing the vascular status in this patient is problematic. To identify the pathology a catheter angiogram was required, so repetition of this study is not considered prudent. Doppler studies could be helpful if re-cannualization of one or more arteries occurs. A three phase nuclear scanmay allow quantification of vascular perfusion.

\section{Conclusion}

The development of severe acral cyanosis, attendant ischemia, and possible threat of necrosis generally triggers a differential diagnosis of 1) peripheral vascular disease, 2) embolic phenomena, 3) hypercoaguable state, thrombotic vasculopathy, 4) vasospastic disease/Reynaud's and 5) vasculitis. The first four can be evaluated with physical exam, noninvasive imaging, and laboratory testing. Localized vasculitis requires tissue biopsy which must be placed in context with the patient's history. When the involved region is distal, such as the foot or hand, obtaining a suitable biopsy can be difficult and nondiagnostic or misleading if too superficial. Since CPAN is hypothesized to be caused by local deposition of antigen antibody complexes of IgM antiphosphatidylserine and prothrombin in the arterial wall causing a necrotizing, segmental leukocytoclastic vasculitis, a targeted therapy with rituximab could be chosen with a high level of confidence of benefit. While selection of appropriate therapy is always important, it was critical in this case with the severity of vas cular compromise and the threat of loss of digit or limb by ischemic necrosis. Consideration was also given to the long term adverse effects of cyclophosphamide, as well as, the increased complexity and frequency of therapy. The use of rituximab affords the chance of reducing or eliminating the inciting $B$ cell line with the intention of altering the typical chronic, relapsing course of CPAN. In the patient presented, recurrence could cause loss of digit or limb. It is the authors' opinion that CPAN should be treated more aggressively from initial diagnosis to prevent morbidity and potentially abort a chronic, relapsing course.

\section{List of abbreviations}

ANA: Antinuclear antibodies

ENA: Extractable nuclear antigens; includes Anti-smith antibody, Ro and La antibodies, Jo1 antibodies, Scl70 antibodies, and ribonuclear protein antibodies

ANCA: Anti-neutrophilic cytoplasmic antibodies; includes $c$ and $p$ ANCA, myeloperoxidase, and protein 3

\section{Competing interests}

The authors declare that they have no competing interests.

\section{Authors' contributions}

\begin{tabular}{|l|c|c|}
\hline Authors' contributions & JES & DAM \\
\hline Research concept and design & -- & -- \\
\hline Collection and/or assembly of data & $\checkmark$ & $\checkmark$ \\
\hline Data analysis and interpretation & $\checkmark$ & $\checkmark$ \\
\hline Writing the article & $\checkmark$ & $\checkmark$ \\
\hline Critical revision of the article & $\checkmark$ & $\checkmark$ \\
\hline Final approval of article & $\checkmark$ & $\checkmark$ \\
\hline Statistical analysis & -- & -- \\
\hline
\end{tabular}

\section{Publication history}

Editor: Antonio G Tristano, Medical Center Carpetana, Spain. Received: 09-Jan-2017 Final Revised: 20-Feb-2017

Accepted: 01-Mar-2017 Published: 11-Mar-2017

\section{References}

1. Jennette JC, Falk RJ, Bacon PA, Basu N, Cid MC, Ferrario F, Flores-Suarez LF, Gross WL, Guillevin L, Hagen EC and Hoffman GS et al. 2012 revised International Chapel Hill Consensus Conference Nomenclature of Vasculitides. Arthritis Rheum. 2013; 65:1-11. | Article | PubMed

2. Mohammad AJ, Jacobsson LT, Mahr AD, Sturfelt $G$ and Segelmark $M$. Prevalence of Wegener's granulomatosis, microscopic polyangiitis, polyarteritis nodosa and Churg-Strauss syndrome within a defined population in southern Sweden. Rheumatology (Oxford). 2007; 46:132937. | Article | PubMed

3. Morgan AJ and Schwartz RA. Cutaneous polyarteritis nodosa: a comprehensive review. Int J Dermatol. 2010; 49:750-6. | Article | PubMed

4. Al-Daraji W, Gregory AN and Carlson JA. "Macular arteritis": a latent form of cutaneous polyarteritis nodosa? Am J Dermatopathol. 2008; 30:145-9. | Article | PubMed

5. Bauza A, Espana A and Idoate M. Case Report Cutaneous Polyarteritis Nodosa. British Journal of Dermatology. 2002; 146:694-699. | Article | PubMed

6. Williams VL, Guirola R, Flemming K, Modi GM, Rosales C and DeGuzman $\mathrm{MM}$. Distal extremity necrosis as a manifestation of cutaneous polyarteritis nodosa: case report and review of the acute management of a pediatric patient. Pediatr Dermatol. 2012; 29:473-8. | Article | PubMed

7. Dhaon P, Bansal N, Das SK, Wakhlu A and Tandon V. Cutaneous polyarteritis nodosa presenting with digital gangrene and breast ulcer. Int J Rheum Dis. 2013; 16:774-6. I Article I PubMed

8. Kawakami T, Yamazaki M, Mizoguchi M and Soma Y. High titer of anti- 
phosphatidylserine-prothrombin complex antibodies in patients with cutaneous polyarteritis nodosa. Arthritis Rheum. 2007; 57:1507-13. | Article I PubMed

9. Kawakami T and Soma Y. Correlation of livedo racemosa, cutaneous inflammatory plaques, and antiphospholipid antibodies in patients with cutaneous polyarteritis nodosa. Medicine (Baltimore). 2011; 90:119-24. | Article | PubMed

10. Fathalla BM, Miller L, Brady S and Schaller JG. Cutaneous polyarteritis nodosa in children. J Am Acad Dermatol. 2005; 53:724-8. | Article | PubMed

11. Maillard H, Szczesniak S, Martin L, Garot D, Machet MC, Machet L, Lorette $\mathrm{G}$ and Vaillant $\mathrm{L}$. [Cutaneous periarteritis nodosa: diagnostic and therapeutic aspects of 9 cases]. Ann Dermatol Venereol. 1999; 126:1259. | PubMed

12. Boehm I and Bauer R. Low-dose methotrexate controls a severe form of polyarteritis nodosa. Arch Dermatol. 2000; 136:167-9. | Article | PubMed

13. Lobo I, Ferreira M, Silva E, Alves R and Selores M. Cutaneous polyarteritis nodosa treated with intravenous immunoglobulins. J Eur Acad Dermatol Venereol. 2008; 22:880-2. | Article | PubMed

14. Krishnan S, Bhakuni DS and Kartik S. Letter to Editor Rituximab in refractory cutaneous polyarteritis. Inter Jour Rheumatic Diseases. 2012; 15:127. | Article

15. Clowse ME, Behera MA, Anders CK, Copland S, Coffman CJ, Leppert $\mathrm{PC}$ and Bastian LA. Ovarian preservation by GnRH agonists during chemotherapy: a meta-analysis. J Womens Health (Larchmt). 2009; 18:311-9. | Article | PubMed Abstract | PubMed FullText

16. Miloslavsky EM, Specks U, Merkel PA, Seo P, Spiera R, Langford CA, Hoffman GS, Kallenberg CG, St Clair EW, Tchao NK, Viviano L, Ding L, Ikle $D$ and Villarreal $M$ et al. Rituximab for the treatment of relapses in antineutrophil cytoplasmic antibody-associated vasculitis. Arthritis Rheumatol. 2014; 66:3151-9. | Article | PubMed Abstract | PubMed FullText

17. Niles J. Rituximab in induction therapy for anti-neutrophil cytoplasmic antibody (ANCA) vasculitis. Clin Exp Immunol. 2011; 164 Suppl 1:27-30. | Article | PubMed Abstract | PubMed FullText

18. Alba MA and Flores-Suarez LF. Rituximab as maintenance therapy for ANCA associated vasculitis: how, when and why? Reumatol Clin. 2016; 12:39-46. | Article | PubMed

\section{Citation:}

Stephan JE and Minna DA. Refractory cutaneous polyarteritis nodosa with threat of loss of feet: successful treatment with rituximab. J Rheumatol Orthop. 2017; 4:1.

http://dx.doi.org/10.7243/2055-7000-4-1 